\title{
Produção de geopolímeros à base de cinza volante usando soluções ativadoras com diferentes composições de $\mathrm{Na}_{2} \mathrm{O}$ e $\mathrm{Na}_{2} \mathrm{SiO}_{3}$
}

\section{(Production of fly ash-based geopolymers using activator solutions with different $\mathrm{Na}_{2} \mathrm{O}$ and $\mathrm{Na}_{2} \mathrm{SiO}_{3}$ compositions)}

\author{
A. G. de S. Azevedo, K. Strecker, A. G. de Araújo Jr., C. A. da Silva \\ Universidade Federal de S. João del-Rei, Campus Sto. Antônio, Pr. Frei Orlando 170, S. João del-Rei, MG, \\ Brasil 36307-352
}

\begin{abstract}
Resumo
Geopolímeros são uma nova classe de ligantes inorgânicos que possuem alta resistência mecânica, inércia química e podem ser fabricados a partir de uma série de subprodutos da indústria. Nesse trabalho amostras de geopolímeros foram produzidas pela ativação da cinza volante proveniente do complexo termelétrico de Jorge Lacerda, SC. Diferentes ativadores alcalinos foram utilizados para a obtenção dos materiais com o intuito de se observar como a modificação da composição química das soluções influencia as propriedades finais dos geopolímeros. Os resultados obtidos demonstraram que os materiais possuem alta resistência à compressão nas primeiras horas de cura $(28 \mathrm{MPa})$ e que aumenta para valores próximos de $48 \mathrm{MPa}$ após 28 dias. Por meio da difração de raios $\mathrm{X}$ observou-se que tanto as cinzas originais quanto as amostras ativadas apresentaram fases minerais comumente encontradas nesse tipo de material. A espectroscopia no infravermelho demonstrou que em todas as amostras, independente da composição química da solução alcalina, modificações nas bandas originais encontradas na cinza volante foram observadas. Tais modificações estão diretamente envolvidas com o processo de geopolimerização. As propriedades físicas das amostras, tais como absorção de água, porosidade e permeabilidade, também tiveram modificações quando diferentes composições químicas foram utilizadas para se ativar as cinzas volantes.
\end{abstract}

Palavras-chave: geopolímeros, cimento geopolimérico, cinza volante, ativação alcalina.

Abstract

Geopolymers are a new class of binder with high mechanical strength, chemical inertia and can be made of by-products. In this work fly ash from a Brazilian power station was used to produce the geopolymer cement. To produce the geopolymers, different activator solutions were used in order to study the influence of the chemical composition of these solutions on the production of the fly ash-based geopolymers. The results showed that the Brazilian fly ash has the potential to be used in the geopolymerization process. The specimens presented high compression strength, $28 \mathrm{MPa}$ after $24 \mathrm{~h}$ and $48 \mathrm{MPa}$ after 28 days of cure. The X-ray diffraction of the original fly ash and the geopolymers samples exhibited some mineral phases presents in the fly ash and new zeolitic phases formed after the geopolymerization process. The infrared spectroscopic of all samples showed some modifications of absorption bands besides new bands corresponding to the geopolymerization process. The physical properties, such as the water absorption, porosity and permeability, changed when the activator solution changed and the curing time was modified.

Keywords: geopolymers, geopolymeric cement, fly ash, alkaline activation.

\section{INTRODUÇÃO}

No atual cenário de crescimento em que nos encontramos, o consumo do cimento Portland vem aumentando ano após ano, representando atualmente um consumo de aproximadamente 2600 milhões de toneladas/ano e com previsão de aumento de quatro vezes nos próximos 40 anos [1]. Atrelado ao aumento do consumo de cimento Portland (CP) existe a preocupação com a emissão de $\mathrm{CO}_{2}$ que ocorre durante a produção do cimento. De acordo com [2], aproximadamente $5 \%$ das emissões globais de $\mathrm{CO}_{2}$ são provenientes da produção do CP. Devido a esses problemas ambientais a busca por novos ligantes capazes de substituir o cimento Portland se faz necessária. Essa nova classe de materiais deve ser capaz de substituir o cimento tradicional e desempenhar o seu mesmo papel, garantindo elevada resistência mecânica, inércia química e também se apresentar com baixo custo para que o mesmo seja competitivo no mercado consumidor $[3,4]$.

Uma saída para o problema ambiental causado pela produção do $\mathrm{CP}$ e, também, para o consumo energético atrelado à sua produção é a utilização de um novo sistema de ligantes. A produção de cimentos álcali-ativados se apresenta como um grande candidato a substituir o cimento Portland nas mais diversas áreas da construção civil [5]. Essa nova classe de ligantes, que é conhecida também como polímeros inorgânicos ou geopolímeros, foi descrita pela primeira vez por Glukhovsky em 1959, ano em que a teoria-base da ativação alcalina se desenvolveu. Em meados da década de 70 o termo geopolímero foi pela primeira vez citado por 
Joseph Davidovits [5, 6]. Glukhovsky em 1957 iniciou em Kiev, Ucrânia, os seus estudos acerca da álcali-ativação de materiais contendo baixos teores de cálcio e elevados teores de silício e alumínio. Seu trabalho foi inovador, pois, até então, somente materiais com altos teores de cálcio tinham sido investigados, como a escória granulada de alto forno [7]. Os materiais utilizados para a obtenção dos geopolímeros podem ser compostos industrializados (metacaulim) ou subprodutos provenientes de processos industriais, como, por exemplo, a cinza volante e escória de alto forno. A utilização de cinzas volantes para a produção dos ligantes geopoliméricos tem sido investigada por vários pesquisadores devido à sua grande produção e sua pouca utilização, tornando-se um problema de armazenamento. Milhares de toneladas de cinza volante são produzidos anualmente e representam um problema ambiental e de saúde pública, devido, principalmente, a processos de percolação e problemas respiratórios, respectivamente.

A nomenclatura usada para nomear os geopolímeros é baseada no termo polissialato, utilizado para abreviar sílico-oxo-aluminato [3, 8, 9], e possui fórmula empírica dada por:

$$
\mathrm{Mn}\left[-\left(\mathrm{Si}-\mathrm{O}_{2}\right)_{\mathrm{z}}-\mathrm{Al}-\mathrm{O}\right]_{\mathrm{n}} \cdot \mathrm{wH}_{2} \mathrm{O}
$$

onde, $\mathrm{M}$ é um cátion (metal alcalino ou metal alcalinoterroso) e $\mathrm{n}$ é o grau de polimerização. O símbolo (-) é usado para demonstrar a presença de uma ligação e $\mathrm{z}$ pode ser 1,2 ou 3. A variação de $\mathrm{z}$ causa variação na razão $\mathrm{SiO}_{2} / \mathrm{Al}_{2} \mathrm{O}_{3}$, que favorece a formação de diferentes tipos de geopolímeros, como resumido na Tabela I; w corresponde ao grau de hidratação do polímero inorgânico [4, 10-12]. Como apresentado na Tabela I, a modificação da composição química no sistema durante a produção dos geopolímeros promove a modificação da unidade repetidora e consequentemente as propriedades do geopolímero. $\mathrm{O}$ foco desse trabalho foi a obtenção de amostras de geopolímeros à base de cinza volante $(\mathrm{CV})$ ativadas com soluções simples e compostas (contendo apenas $\mathrm{NaOH}$ e $\mathrm{NaOH}+\mathrm{Na}_{2} \mathrm{SiO}_{3}$,

Tabela I - Monômeros-base de formação dos geopolímeros. [Table I - Monomers of the geopolymers formation.]

\begin{tabular}{cc}
\hline Monômero & $\begin{array}{c}\text { Razão } \\
\text { Si/Al }\end{array}$ \\
\hline Poli(sialato) (-Si-O-Al-O-) & $1: 1$ \\
Poli(sialato-siloxo) (-Si-O-Al-O-Si-O-) & $2: 1$ \\
Poli(sialato-dissiloxo) (-Si-O-Al-O-Si-O-Si-O-) & $3: 1$ \\
\hline
\end{tabular}

respectivamente), com estudo da influência da modificação da composição química das soluções e as variações da estrutura dos geopolímeros produzidos. O intuito foi produzir uma formulação para a fabricação de geopolímeros à base de CV com elevada resistência mecânica podendo, consequentemente, substituir o cimento Portland na indústria da construção civil.

\section{MATERIAIS E MÉTODOS}

Cinza volante $(\mathrm{CV})$ : foi utilizada uma $\mathrm{CV}$ proveniente do complexo termelétrico de Jorge Lacerda, situado na cidade de Capivari de Baixo no estado de Santa Catarina/Brasil. A cinza foi adquirida da empresa PozoFly Comércio de Cinzas Lima, que recebeu a cinza na forma bruta e realizou processos de armazenamento e moagem para posterior distribuição. Silicato de sódio alcalino $\left(\mathrm{Na}_{2} \mathrm{SiO}_{3}\right)$ : foi utilizado para a confecção das soluções ativadoras (SA) adquirido da Diatom, cód. R 2252, e apresentou a composição fornecida pelo fabricante (Tabela II). Hidróxido de sódio $(\mathrm{NaOH})$ : as soluções alcalinas foram fabricadas com hidróxido de sódio do tipo P.A., Sulfal Química, pureza de 98\%. A escolha do ativador alcalino foi devido ao menor preço de mercado quando comparado ao $\mathrm{KOH}$.

Métodos: para a produção dos geopolímeros (GP), a cinza volante foi misturada com as soluções ativadoras em temperatura ambiente e a pasta obtida foi submetida à cura térmica por $24 \mathrm{~h} \mathrm{a} 65^{\circ} \mathrm{C}$. A composição química dos ativadores utilizados está apresentada na Tabela III. Os ativadores alcalinos foram preparados com $24 \mathrm{~h}$ de antecedência para se evitar aquecimento durante a dissolução do $\mathrm{NaOH}$, o que poderia afetar as reações de geopolimerização. Em todas as misturas a razão entre a solução ativadora e a cinza volante (SA/CV) utilizada foi mantida constante em valor próximo de 0,5. As pastas frescas foram adicionadas a moldes plásticos com dimensões de $50 \mathrm{~mm}$ x $25 \mathrm{~mm}$ (altura $\mathrm{x}$ diâmetro) e seladas utilizando um filme plástico para evitar a perda de água por evaporação. Os moldes foram vibrados em mesa vibratória por $60 \mathrm{~s}$ para remover as bolhas formadas durante o preenchimento dos moldes. A nomenclatura utilizada para identificar as amostras consistiu em apresentar a concentração de $\mathrm{NaOH}\left(\mathrm{mol} . \mathrm{L}^{-1}\right)$ das soluções ativadoras, dada pela letra $\mathrm{H}$, e também a porcentagem de $\mathrm{Na}_{2} \mathrm{SiO}_{3}$ utilizada em cada mistura (\% em massa), representada pela letra S. O tempo de cura foi expresso pelo número logo à frente da composição da solução ativadora utilizada. Como exemplo, pode-se citar a amostra H12S20-7, que correspondeu à amostra produzida utilizando a solução de $\mathrm{NaOH}(12 \mathrm{M})$ e com $20 \%$ de $\mathrm{Na}_{2} \mathrm{SiO}_{3}$ após 7 dias de cura.

Tabela II - Composição e propriedades físicas do silicato alcalino fornecidas pelo fabricante. [Table II - Composition and physical properties of alkaline silicate.]

\begin{tabular}{ccccccc}
\hline & Composição (\%) & & Total de sólidos \\
$(\%)$ & $\mathrm{Na}_{2} \mathrm{O}$ & $\mathrm{SiO}_{2}$ & $\mathrm{SiO}_{2} / \mathrm{Na}_{2} \mathrm{O}$ & $\begin{array}{c}\text { Umidade } \\
(\%)\end{array}$ & $\begin{array}{c}\text { Densidade a } 25{ }^{\circ} \mathrm{C} \\
\left(\mathrm{g} / \mathrm{cm}^{3}\right)\end{array}$ & $\begin{array}{c}\text { Viscosidade } \\
(\mathrm{cP})\end{array}$ \\
\hline 14,98 & 33,57 & 2,40 & 48,55 & 51,44 & 1,58 & 1,35 \\
\hline
\end{tabular}


Tabela III - Informações acerca das soluções alcalinas (SA) utilizadas para produzir os geopolímeros à base de cinza volante (CV). [Table III - Information about the alkaline solutions used to produce the fly ash (CV) based geopolymeric samples.]

\begin{tabular}{ccccccc}
\hline Amostra & {$[\mathrm{NaOH}]\left(\mathrm{mol}^{-L^{-1}}\right)$} & $\% \mathrm{Na}_{2} \mathrm{SiO}_{3}{ }^{\mathrm{a}}$ & $\% \mathrm{Na}_{2} \mathrm{O}^{\mathrm{b}}$ & $\% \mathrm{SiO}_{2}{ }^{\mathrm{b}}$ & $\mathrm{SiO}_{2} / \mathrm{Na}_{2} \mathrm{O}^{\mathrm{b}}$ & $\mathrm{H}_{2} \mathrm{O} / \mathrm{CV}$ \\
\hline $\mathrm{H} 12 \mathrm{~S} 0$ & 12 & 0 & 13,51 & 0 & 0 & 0,33 \\
$\mathrm{H} 12 \mathrm{~S} 10$ & 12 & 10 & 12,31 & 3,36 & 0,27 & 0,31 \\
$\mathrm{H} 12 \mathrm{~S} 20$ & 12 & 20 & 11,11 & 6,71 & 0,60 & 0,30 \\
$\mathrm{H} 12 \mathrm{~S} 30$ & 12 & 30 & 9,90 & 10,07 & 1,02 & 0,28 \\
\hline
\end{tabular}

${ }^{a}$ - em relação à quantidade total de solução (em massa); ${ }^{b}$ - em relação à quantidade de CV adicionada.

Cinco corpos de prova de cada condição foram utilizados para as medidas das resistências à compressão. Para as análises microestruturais foram utilizados fragmentos dos corpos de prova fraturados, que foram quebrados, macerados em moinho de ágata e peneirados em peneira de 100 mesh. As amostras foram caracterizadas por meio da compressão uniaxial como descrito na norma NBR 5739 [13], utilizando uma máquina universal da Shimadzu, AG-X Plus. Os dados obtidos foram tratados por meio da utilização do software Trapezium X, v. 1.2.6, Shimadzu. O módulo de elasticidade estático foi calculado por intermédio da curva tensãodeformação gerada pelo aparelho, obtido pelo módulo tangente após o ajuste da inclinação da curva pelo software.

A composição química da cinza volante foi obtida utilizando um equipamento da Shimadzu (energy dispersive $X$-ray fluorescence spectrometer, EDX). A massa específica da cinza volante utilizada foi obtida por um picnômetro a hélio da Micromeritics, AccuPyc 1340. A granulometria foi obtida utilizando um aparelho da Malvern, Mastersizer $2000(0,02$ a $2000 \mu \mathrm{m})$. As cinzas foram dispersas em água deionizada e as condições de ensaio foram: agitação de 1500 rpm, tempo de ultrassom de 2,5 min, obscuração entre $10 \mathrm{e}$ $20 \%$ e tempo de dispersão de $5 \mathrm{~min}$. A caracterização das fases cristalinas da cinza volante e das pastas álcali-ativadas foi realizada por meio da utilização de um difratômetro da Shimadzu, XRD-6000, com tensão de $30 \mathrm{kV}$, corrente de $30 \mathrm{~mA}$, radiação $\mathrm{CuK} \alpha(\lambda=0,15462 \mathrm{~nm})$ com passo de $0,02^{\circ}$ e velocidade de varredura de $2 \% \mathrm{mim}$, entre 5 e $60^{\circ}$. A identificação das fases foi realizada por comparação dos picos de difração obtidos com os fornecidos pelos arquivos do JCPDS (banco de dados de estruturas cristalinas inorgânicas). As análises espectroscópicas foram realizadas em um espectroscópio da Perkim Elmer, Sprectrum 1000. As amostras foram analisadas na forma de pastilhas misturando a CV e o GP com $\mathrm{KBr}$ de elevada pureza (grau espectroscópico) e aplicando uma carga de 10 ton por 60 s. As análises foram obtidas a partir da média de 16 ciclos de varredura, na faixa de número de onda de 400 a 4000 $\mathrm{cm}^{-1}$ e resolução de $4 \mathrm{~cm}^{-1}$. Para a análise da morfologia das amostras de cinza volante e dos produtos da álcali-ativação foi utilizado um microscópio eletrônico de varredura da Hitachi, TM 300, com espectrômetro por dispersão de energia (EDS) acoplado da Bruker, X-Flash Mim SVE. As propriedades físicas dos geopolímeros obtidos foram obtidas utilizando a norma britânica BS EN ISO 10545-3 (1997).

\section{RESULTADOS E DISCUSSÃO}

\section{Caracterização da cinza volante}

Fluorescência de raios $X$ (FRX): a composição da cinza volante $(\mathrm{CV})$ utilizada para a obtenção dos corpos de prova é apresentada na Tabela IV. A CV foi composta basicamente de sílica e alumina; entretanto, existiu a presença de outros óxidos em menores quantidades. A cinza volante foi do tipo F, de acordo com a norma ASTM C618 (2010) que classifica como cinzas volantes desse tipo o material que apresenta um teor de $\mathrm{SiO}_{2}, \mathrm{Al}_{2} \mathrm{O}_{3}$ e $\mathrm{Fe}_{2} \mathrm{O}_{3}$ maior do que $70 \%$.

Análise granulométrica por difração de laser $e$ picnometria: a Fig. 1 apresenta os dados relacionados à granulometria da cinza volante. Os tamanhos das partículas variaram entre 0,275 e $209 \mu \mathrm{m}$. A Tabela V apresenta os diâmetros D10, D50 e D80, que são os tamanhos de partículas abaixo dos quais correspondem a 10,50 e $80 \%$ da massa do material, respectivamente. Na Tabela $V$ também se encontra a massa específica da cinza volante obtida por meio da picnometria a gás.

Difração de raios $X(D R X)$ : o difratograma da cinza volante (Fig. 2a) apresenta vários picos de difração de fases cristalinas que podem estar presentes no carvão ou podem se formar durante a queima dos mesmos nas usinas termelétricas [14]. Picos de difração correspondentes a fases cristalinas de quartzo - $\mathrm{SiO}_{2}$ (ICSD-100341), mulita - $\mathrm{Al}_{6} \mathrm{Si}_{2} \mathrm{O}_{13}$ (ICSD-66447, 66448, 66449) e hematita - $\mathrm{Fe}_{2} \mathrm{O}_{3}$ (ICSD-82137) foram identificados na CV. O difratograma da cinza também apresentou uma banda larga (halo) entre

Tabela IV - Composição química (\% em massa) da cinza volante utilizada para a produção de geopolímeros.

[Table IV - Chemical composition (wt\%) of the fly ash used to produce the geopolymers.]

\begin{tabular}{ccccccccccc}
\hline $\mathrm{SiO}_{2}$ & $\mathrm{Al}_{2} \mathrm{O}_{3}$ & $\mathrm{Fe}_{2} \mathrm{O}_{3}$ & $\mathrm{~K}_{2} \mathrm{O}$ & $\mathrm{CaO}$ & $\mathrm{TiO}_{2}$ & $\mathrm{SO}_{3}$ & $\mathrm{ZrO}_{2}$ & $\mathrm{~V}_{2} \mathrm{O}_{5}$ & Outros óxidos* & $\mathrm{SiO}_{2} / \mathrm{Al}_{2} \mathrm{O}_{3}$ \\
\hline 56,03 & 29,41 & 6,16 & 3,77 & 2,07 & 1,81 & 0,26 & 0,11 & 0,10 & 0,77 & 3,88 \\
\hline
\end{tabular}

* - $\mathrm{ZnO}, \mathrm{MnO}, \mathrm{Cr}_{2} \mathrm{O}_{3}, \mathrm{SrO}, \mathrm{CuO}, \mathrm{Rb}_{2} \mathrm{O}, \mathrm{Y}_{2} \mathrm{O}_{3}, \mathrm{PbO}, \mathrm{Ga}_{2} \mathrm{O}_{3}, \mathrm{GeO}_{2}, \mathrm{NiO}$ e NbO. 


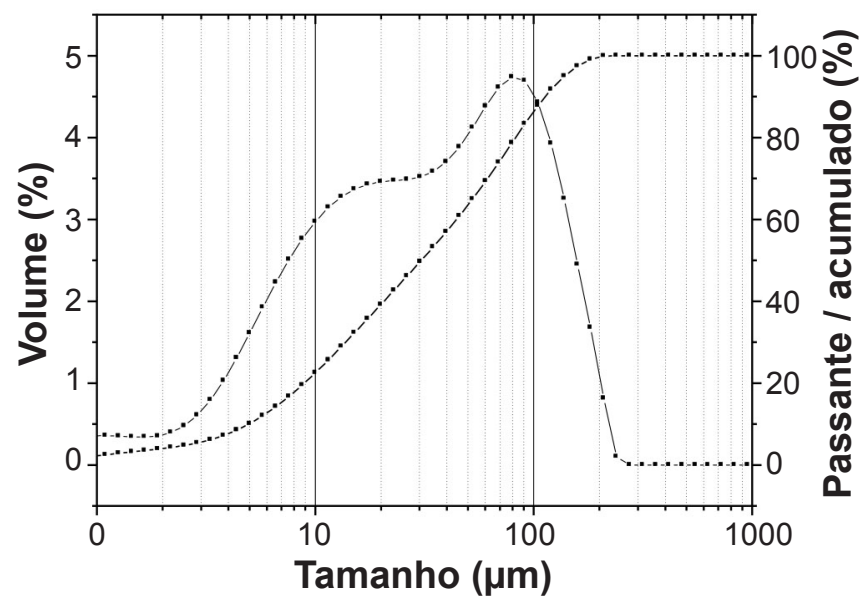

Figura 1: Curvas de distribuição granulométrica da cinza volante. [Figure 1: Particle size distribution curves of the fly ash.]

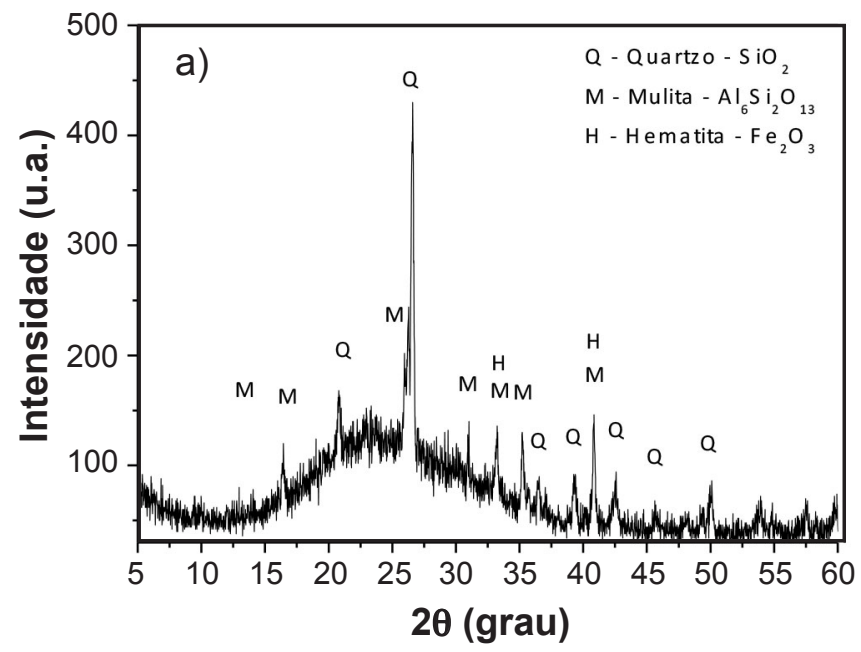

18 e $35^{\circ}$ que confirmou a presença de aluminossilicatos amorfos que se dissolvem durante a ativação da cinza com as soluções alcalinas [14].

Espectroscopia no infravermelho com transformada de Fourier (IVTF): a Fig. 2b apresenta as bandas de absorção no infravermelho da CV. As bandas em 3467 e $1641 \mathrm{~cm}^{-1}$ foram relacionadas ao estiramento assimétrico das moléculas de

Tabela V - Tamanhos de partículas (D10, D50 e D80) e massa específica da cinza volante.

[Table V-Particle sizes (D80, D50 and D10) and specific mass of fly ash.]

\begin{tabular}{ccc}
\hline Diâmetro & Tamanho $(\mu \mathrm{m})$ & Massa específica $\left(\mathrm{g} / \mathrm{cm}^{3}\right)$ \\
\hline D10 & 4,99 & \\
D50 & 30,82 & $2,21 \pm 0,01$ \\
D80 & 115,12 & \\
\hline
\end{tabular}

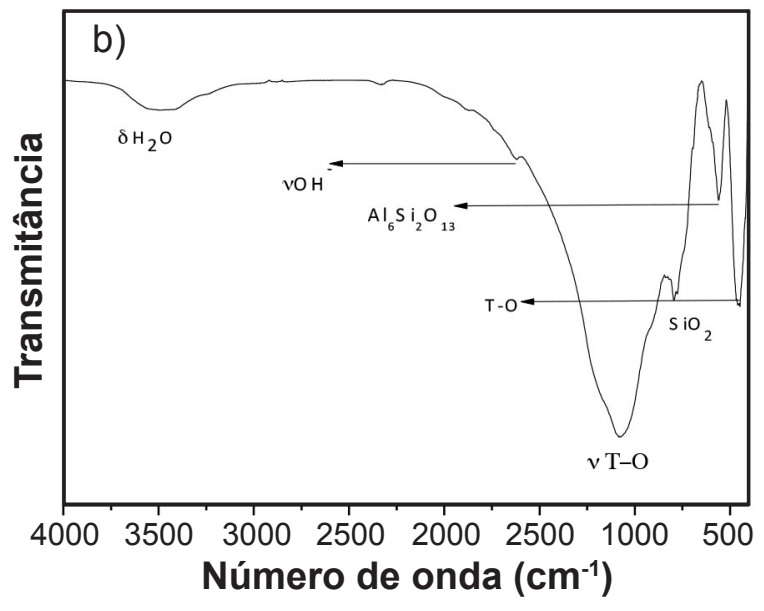

Figura 2: Difratograma de raios X (a) e espectro de IVTF (b) da cinza volante utilizada.

[Figure 2: X-ray diffraction pattern (a) and FTIR spectrum (b) of the fly ash.]
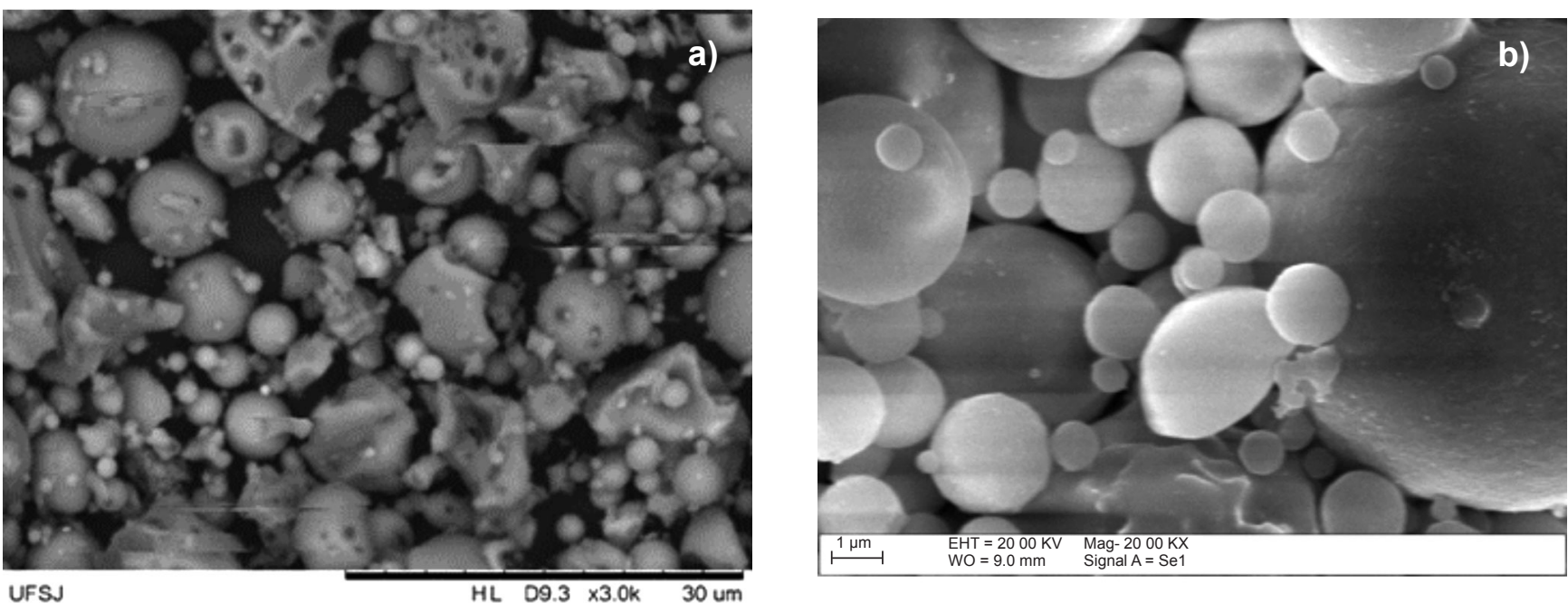

Figura 3: Micrografias obtidas por microscopia eletrônica de varredura da cinza volante utilizada.

[Figure 3: SEM micrographs of the fly ash.] 
Tabela VI - Resultados da análise por EDS da cinza volante. [Table VI - Results of EDS analysis of the fly ash.]

\begin{tabular}{ccc}
\hline Elemento & \% em massa & \% atômica \\
\hline Oxigênio & 48,23 & 63,13 \\
Silício & 33,37 & 24,88 \\
Alumínio & 10,29 & 7,99 \\
Potássio & 5,92 & 3,17 \\
Ferro & 2,19 & 0,82 \\
Total & 100 & 100 \\
\hline
\end{tabular}

$\mathrm{H}_{2} \mathrm{O}$ fracamente adsorvidas na superfície e à deformação do grupamento $\mathrm{OH}$, respectivamente. Bandas correspondentes ao $\mathrm{SiO}_{2}$ e $\mathrm{Al}_{6} \mathrm{Si}_{2} \mathrm{O}_{13}$ foram observadas em comprimentos de onda próximos de 794 e $559 \mathrm{~cm}^{-1}$. De acordo com [15], as bandas próximas de 1090 e $458 \mathrm{~cm}^{-1}$ foram associadas ao estiramento e às vibrações de flexão do grupo $\mathrm{T}-\mathrm{O}(\mathrm{T}=\mathrm{Si}$ e $\mathrm{Al})$, respectivamente.

Microscopia eletrônica de varredura (MEV/EDS): a morfologia da cinza volante está apresentada na Fig. 3. É possível notar que o material foi composto de partículas esféricas e tamanho submicrométrico. Possuiu tamanho aleatório, como apresentado nos resultados da granulometria. As esferas podem ser ocas ou podem conter partículas de menor dimensão no seu interior [16]. A composição da superfície da cinza é apresentada na Tabela VI. A composição química obtida por EDS foi próxima daquela obtida por meio da FRX. Entretanto, deve-se ressaltar que a composição pode variar de uma partícula para outra devido aos processos de queima do carvão mineral que ocorrem nas termelétricas, o que torna a composição dos materiais de partida uma peça fundamental para o melhor entendimento dos processos de geopolimerização [17].

\section{Caracterização dos geopolímeros}

Propriedades mecânicas dos geopolímeros à base de $C V$ : os valores de resistência à compressão uniaxial e do módulo de elasticidade estático das amostras de geopolímeros após 1, 7 e 28 dias estão apresentados na Tabela VII. A resistência mecânica dos geopolímeros foi diretamente relacionada com a composição química da solução ativadora. A modificação dos valores de $\mathrm{Na}_{2} \mathrm{O}$ e $\mathrm{SiO}_{2}$ presentes nas soluções modificaram de forma significativa os valores de resistência mecânica. Os corpos de prova (CPs) produzidos com a composição H12S0 proporcionou a obtenção de resistência à compressão próxima de $19 \mathrm{MPa}$ abaixo daquelas apresentadas pelos CPs produzidos com as soluções compostas $\left(\mathrm{NaOH}+\mathrm{Na}_{2} \mathrm{SiO}_{3}\right)$, cujo valor máximo de 47,6 MPa foi observado para a H12S30 após 28 dias de cura. Esse resultado demonstrou como a adição de uma fonte extra de $\mathrm{SiO}_{2}$ promove o aumento da resistência dos geopolímeros à base de CV. Outro fator que favoreceu o ganho da resistência dos geopolímeros foi a razão $\mathrm{SiO}_{2} / \mathrm{Na}_{2} \mathrm{O}$ presente na solução ativadora utilizada. Nesse trabalho a composição H12S30 apresentou razão mais próxima de 1,0, que, de acordo com $[18,19]$, é o valor ideal para a obtenção de matrizes geopoliméricas de elevada resistência mecânica.

Análise das fases cristalinas: os difratogramas de raios $\mathrm{X}$ da cinza volante e também das amostras de GP fabricadas com diferentes soluções alcalinas após 28 dias de cura estão apresentados na Fig. 4. É possível observar picos referentes às fases cristalinas encontradas na cinza volante (quartzo,

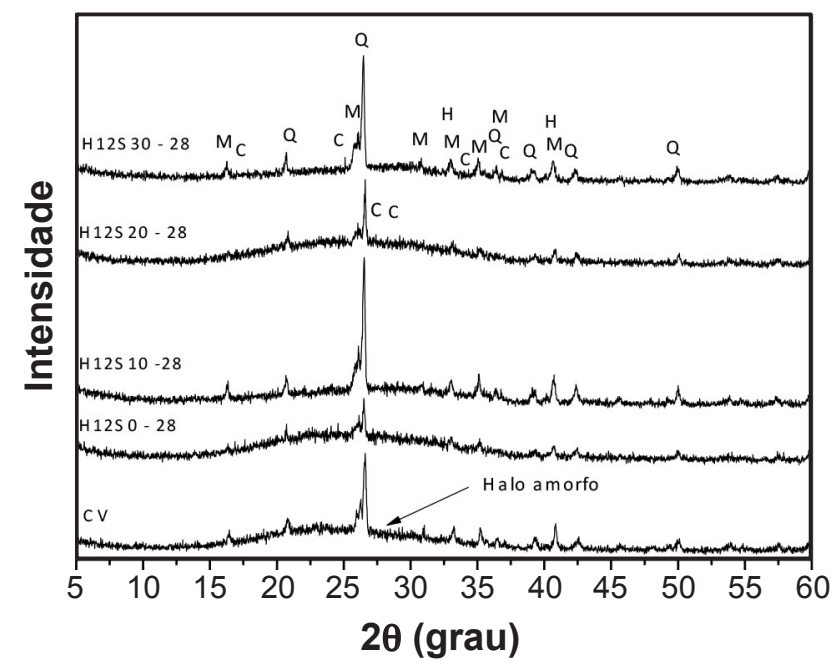

Figura 4: Difratogramas de raios $\mathrm{X}$ da cinza volante original e das amostras de geopolímeros após 28 dias de cura. Q - quartzo, $\mathrm{SiO}_{2} ; \mathrm{M}$ - mulita, $\mathrm{Al}_{6} \mathrm{Si}_{2} \mathrm{O}_{13} ; \mathrm{H}$ - hematita, $\mathrm{Fe}_{2} \mathrm{O}_{3} ; \mathrm{C}$ - Na-chabazita, $\mathrm{NaAlSi}{ }_{2} \mathrm{O}_{6} \cdot 3 \mathrm{H}_{2} \mathrm{O}$.

[Figure 4: X-ray diffraction patterns of the fly ash and geopolymers samples after 28 days of cure. $Q$ - quartz, $\mathrm{SiO}_{2} ; \mathrm{M}$ - mullite, $\mathrm{Al}_{6} \mathrm{Si}_{2} \mathrm{O}_{13} ; \mathrm{H}$-hematite, $\mathrm{Fe}_{2} \mathrm{O}_{3}$; $\mathrm{C}$ - Na-chabazite, $\mathrm{NaAlSi}_{2} \mathrm{O}_{6} 3 \mathrm{H}_{2} \mathrm{O}$.]

Tabela VII - Valores de resistência a compressão e módulo de elasticidade dos geopolímeros à base de cinza volante em diferentes tempos de cura.

[Table VII - Compressive strength and elastic modulus of the fly ash based geopolymers after different curing times.]

\begin{tabular}{ccccccc}
\hline \multirow{2}{*}{ Amostra } & \multicolumn{3}{c}{ Resistência à compressão $(\mathrm{MPa})$} & \multicolumn{3}{c}{ Módulo de elasticidade (GPa) } \\
& 1 dia & 7 dias & 28 dias & 1 dia & 7 dias & 28 dias \\
\hline H12S0 & $9,8 \pm 1,6$ & $10,8 \pm 1,4$ & $19,1 \pm 1,1$ & $1,2 \pm 0,3$ & $1,2 \pm 0,3$ & $2,6 \pm 0,2$ \\
H12S10 & $38,5 \pm 1,5$ & $38,7 \pm 1,3$ & $33,8 \pm 1,2$ & $4,2 \pm 0,2$ & $4,4 \pm 0,1$ & $4,0 \pm 0,1$ \\
H12S20 & $28,3 \pm 2,7$ & $33,3 \pm 4,0$ & $39,2 \pm 1,6$ & $3,2 \pm 0,3$ & $3,6 \pm 0,4$ & $4,2 \pm 0,4$ \\
H12S30 & $27,8 \pm 3,7$ & $48,2 \pm 2,1$ & $47,6 \pm 4,5$ & $3,0 \pm 0,2$ & $3,9 \pm 0,4$ & $4,5 \pm 0,2$ \\
\hline
\end{tabular}


mulita e hematita). A observação desses picos nas amostras de GP demonstra que as fases de elevada ordenação atômica não foram dissolvidas durante os processos de geopolimerização. Entretanto, o halo correspondente à fase amorfa da cinza volante presente entre 18 e $35^{\circ}$ se deslocou para angulações próximas de 18 e $40^{\circ}$, o que, de acordo com [16], confirmou a formação do gel aluminossilicato amorfo, que é o responsável pelo ganho de resistência mecânica das pastas geopoliméricas. Com a adição de $\mathrm{Na}_{2} \mathrm{SiO}_{3}$ no sistema foi possível observar picos correspondentes à fase Na-chabazita, $\mathrm{NaAlSi}_{2} \mathrm{O}_{6} \cdot 3 \mathrm{H}_{2} \mathrm{O}$ (JCPDS 19-1178). Esse composto é um precursor zeolítico muito identificado em processos de obtenção de zeólitas artificiais [17].

Espectroscopia no infravermelho com transformada de Fourier (IVTF): a técnica de espectroscopia no infravermelho é conhecida por ser capaz de caracterizar materiais com ordenação atômica de curto alcance, como é o caso do polímero inorgânico formado nas amostras. As bandas da $\mathrm{CV}$ original sofreram algumas alterações quando entraram em contato com a solução ativadora (Fig. 5). A banda próxima de 3200 a $3600 \mathrm{~cm}^{-1}$ foi relacionada ao estiramento do grupo O-H presente nas moléculas de água fracamente adsorvidas na superfície [20]. A intensidade dessa banda aumentou com a quantidade de água em cada solução e também com a força com que essas moléculas estão presas à superfície dos geopolímeros à base de CV. A banda próxima de $1650 \mathrm{~cm}^{-1}$ foi relacionada com a flexão dos grupos H-O-H e é um tipo comum de vibração em aluminossilicatos [21]. Com o aumento do tempo de cura as moléculas de água tenderam a deixar a estrutura do material devido ao processo de policondensação e evaporação [22]. A banda próxima de $1100 \mathrm{~cm}^{-1}$ da cinza volante original sofreu um deslocamento para regiões de menores números de onda. Esse deslocamento foi associado com o processo de geopolimerização e é intitulado como a digital do processo de produção dos polímeros inorgânicos. Em todos os espectros foi possível observar uma mudança da posição original que correspondeu a vibrações de estiramento assimétrico do grupo Si-O para regiões próximas de 900 a $1002 \mathrm{~cm}^{-1}$, correspondentes ao grupo Si-O-Al, o que confirmou a troca de átomos de Si por Al na estrutura geopolimérica $[15,22$, 23]. As bandas com números de onda próximos de $1450 \mathrm{e}$ $1647 \mathrm{~cm}^{-1}$ foram relacionadas com a formação de espécies carbonatadas na superfície dos corpos de prova. $\mathrm{O}$ álcali em excesso $\left(\mathrm{Na}_{2} \mathrm{O}\right)$ se difundiu até a superfície e reagiu com o $\mathrm{CO}_{2}$ presente na atmosfera, formando espécies carbonatadas do tipo carbonato de sódio $\left(\mathrm{Na}_{2} \mathrm{CO}_{3}\right)$ e bicarbonato de sódio $\mathrm{NaHCO}_{3}$ [24].

Absorção de água, porosidade, densidade $\mathrm{e}$ permeabilidade: os valores relacionados às propriedades físicas dos geopolímeros são apresentados na Tabela VIII. A absorção de água das amostras variou entre 12,3 e 18,0\% após 28 dias de cura. Entretanto, a menor absorção foi obtida nas amostras fabricadas com a solução H12S30. Esses valores corroboraram os valores de porosidade obtidos para os geopolímeros. A porosidade aparente da amostra H12S30-28 apresentou valor de 20,1\% enquanto a amostra

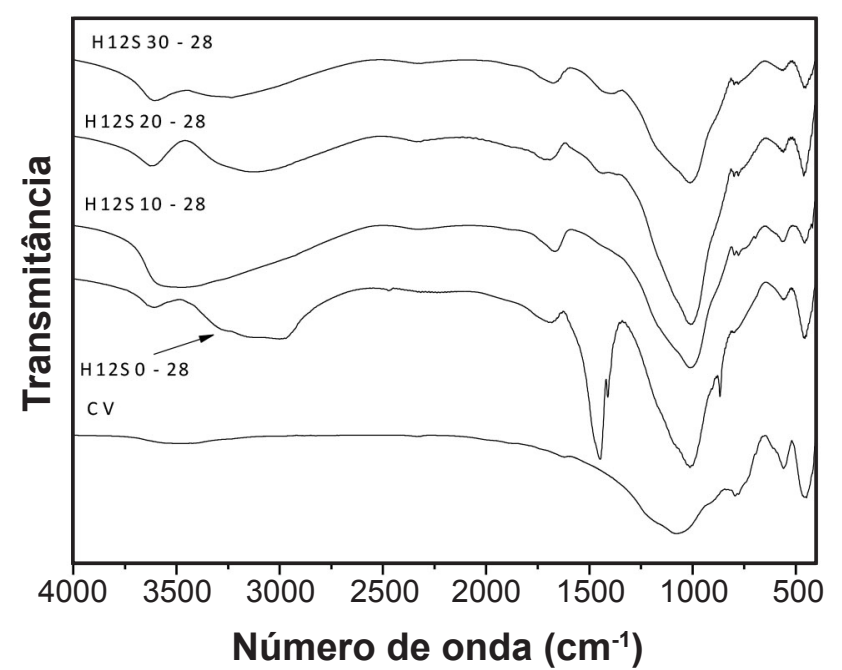

Figura 5: Espectros de IVTF da cinza volante original e das amostras de GP fabricadas com diferentes soluções alcalinas.

[Figure 5: FTIR spectra of the original fly ash and geopolymeric samples made using different alkaline solutions.]

H12S10-28 apresentou 27,2\%. As densidades dos corpos de prova apresentaram valores entre $1,51 \mathrm{e} 1,63 \mathrm{~g} / \mathrm{cm}^{3}$. Esses resultados foram de acordo com os encontrados em [25], em que os autores estudaram as propriedades físicas de geopolímeros à base de CV. A permeabilidade apresentou modificação significativa com a alteração da composição química da solução ativadora. Os CPs produzidos com a solução simples apresentaram valor de permeabilidade elevado quando comparado ao valor dos CPs obtidos com a solução composta, 27,7 e $0,7 \times 10^{-16} \mathrm{~m}^{2}$ para os geopolímeros H12S0-28 e H12S30-28, respectivamente. Esse fato pode estar relacionado com a maior capacidade de formação da estrutura geopolimérica com a adição do silicato de sódio alcalino. Esse resultado demonstrou que o aumento da adição de $\mathrm{Na}_{2} \mathrm{SiO}_{3}$ no sistema promoveu a diminuição da permeabilidade dos corpos de prova, o que favoreceu a obtenção de um sistema ligante mais compacto e com menor porosidade aberta. Demonstrou também que o ajuste da razão $\mathrm{SiO}_{2} / \mathrm{Na}_{2} \mathrm{O}$ em valores próximos de 1,0 favoreceu a obtenção de uma matriz menos permeável e mais densa, o que é desejável em materiais cimentantes.

Microscopia eletrônica de varredura e espectroscopia de energia dispersiva (MEV/EDS): a morfologia e a composição química superficial dos produtos geopolíméricos após 28 dias de cura são apresentados na Fig. 6 e na Tabela IX, respectivamente. As imagens apresentadas diferem das imagens obtidas da CV original. Foi possível observar a formação de um gel rico em aluminossilicato na superfície de todas as amostras e que foi o principal responsável pelo ganho de resistência mecânica dos CPs. As imagens apresentam algumas partículas esféricas que continuaram presentes mesmo depois do contato da $\mathrm{CV}$ com a solução alcalina. Entretanto, na amostra H12S0-28 as esferas se apresentaram intactas, diferente das amostras H12S1028, H12S20-28 e H12S30-28 em que as partículas de CV apresentaram morfologia alterada devido aos processos de 
Tabela VIII - Absorção de água, porosidade aparente, densidade aparente e permeabilidade dos geopolímeros à base de $\mathrm{CV}$ após 28 dias de cura.

[Table VIII - Water absorption, apparent porosity, apparent density and permeability of the fly ash based geopolymers after 28 days of cure.]

\begin{tabular}{ccccc}
\hline Amostra & $\begin{array}{c}\text { Absorção de água } \\
(\%)\end{array}$ & $\begin{array}{c}\text { Porosidade aparente } \\
(\%)\end{array}$ & $\begin{array}{r}\text { Densidade aparente } \\
\left(\mathrm{g} / \mathrm{cm}^{3}\right)\end{array}$ & $\begin{array}{c}\text { Permeabilidade } \\
\left(10^{-16} \mathrm{~m}^{2}\right)\end{array}$ \\
\hline H12S0 & 15,4 & 24,0 & 1,56 & $27,7 \pm 4,9$ \\
H12S10 & 18,0 & 27,2 & 1,51 & $2,0 \pm 0,6$ \\
H12S20 & 16,1 & 25,6 & 1,59 & $1,7 \pm 0,6$ \\
H12S30 & 12,3 & 20,1 & 1,63 & $0,7 \pm 0,6$ \\
\hline
\end{tabular}

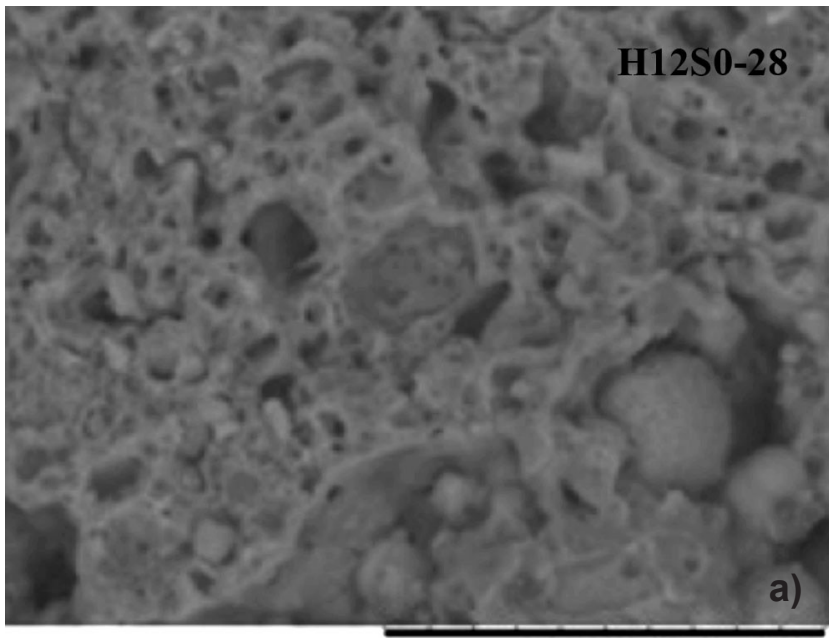

UFSJ

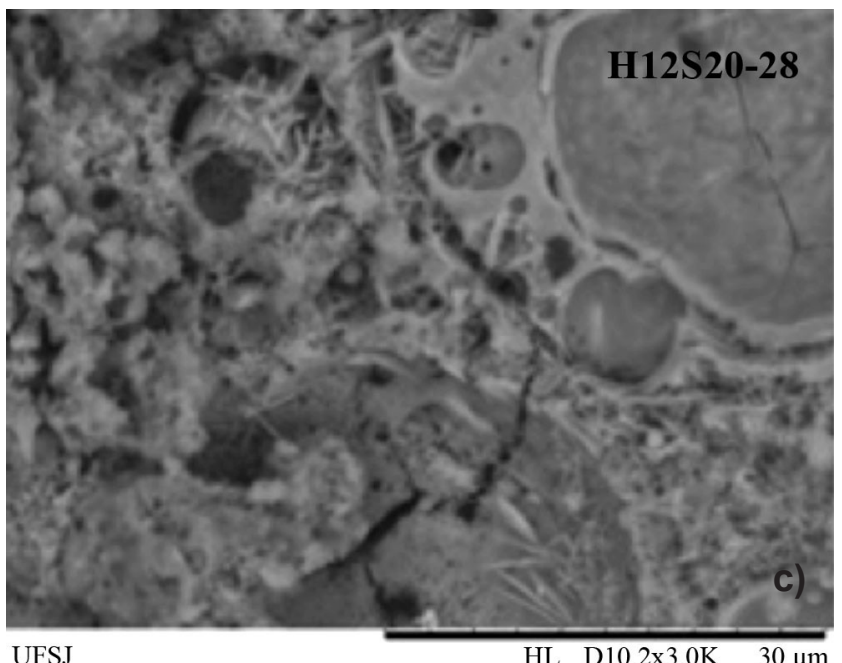

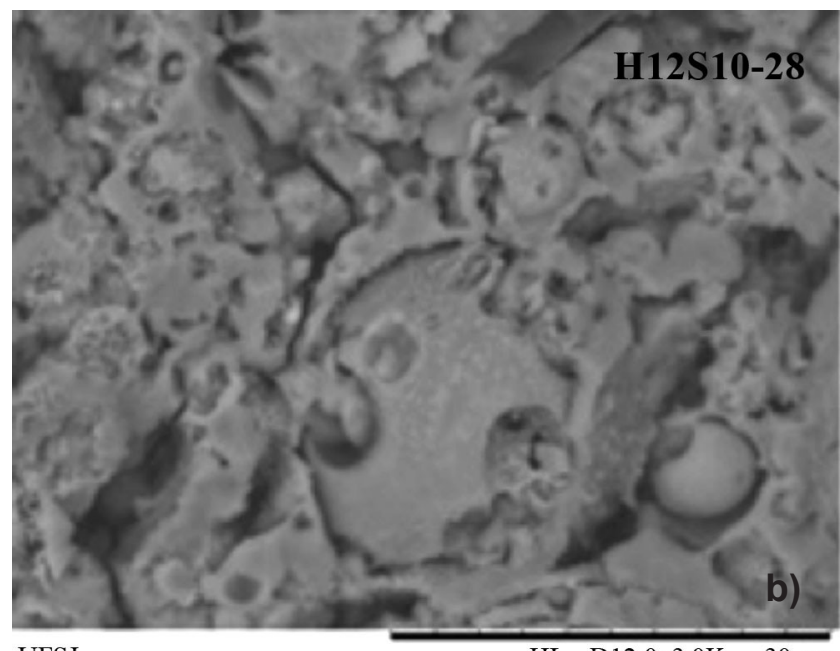

UFS J

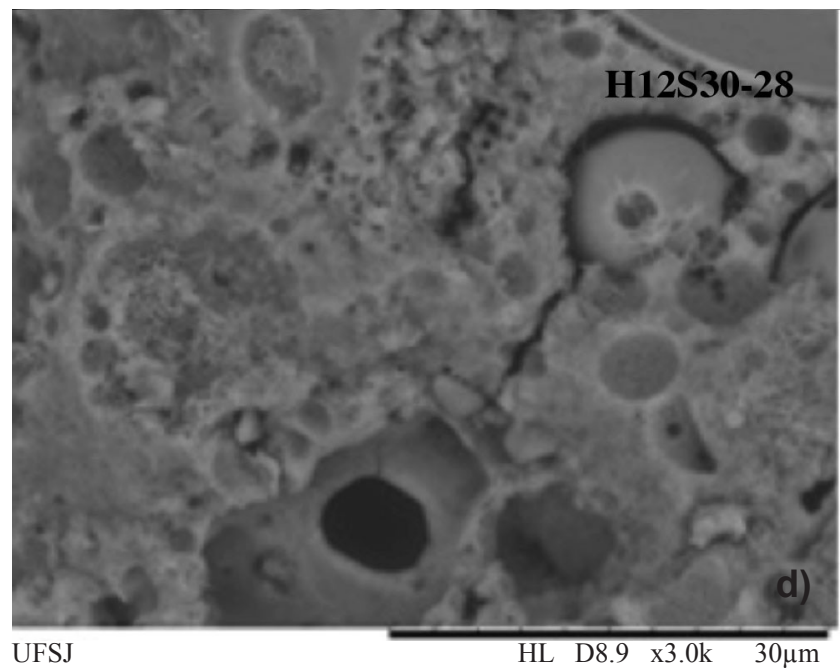

Figura 6: Micrografias obtidas por microscopia eletrônica de varredura dos geopolímeros à base de cinza volante após 28 dias de cura. [Figure 6: SEM micrographs of the fly ash based geopolymers after 28 days of cure.]

dissolução, o que pode estar relacionado com o aumento da razão $\mathrm{SiO}_{2} / \mathrm{Na}_{2} \mathrm{O}$ das soluções. Na amostra H12S20-28 observou-se a formação de estruturas aciculares que foram relacionadas com a reação de produtos alcalinos em excesso com o $\mathrm{CO}_{2}$ atmosférico, como apresentado nos resultados de IVTF. A amostra H12S30-28 apresentou uma morfologia em que o gel formado se encontrou mais densificado e as partículas de CV presentes estavam embebidas no gel de aluminossilicato. A estrutura não apresentou grandes defeitos superficiais tais como microtrincas, o que pode diminuir a resistência mecânica. A morfologia densa observada corroborou os resultados de densidade e permeabilidade em que os CPs fabricados com a solução H12S30 se apresentaram mais densos e com a menor permeabilidade 
Tabela IX - Resultados da análise de EDS das amostras de GP produzidas com diferentes soluções alcalinas após 28 dias de cura.

[Table IX - Results of EDS analysis of the geopolymeric samples made using different alkaline solutions after 28 days of cure.]

\begin{tabular}{cccccc}
\hline Amostra & $\mathrm{Si}(\mathrm{at} \%)$ & $\mathrm{Al}(\mathrm{at} \%)$ & $\mathrm{Na}(\mathrm{at} \%)$ & $\mathrm{Si} / \mathrm{Al}$ & $\mathrm{Na} / \mathrm{Al}$ \\
\hline H12S0 & 48,0 & 19,8 & 27,2 & 2,43 & 1,37 \\
H12S10 & 35,3 & 14,8 & 46,0 & 2,39 & 3,11 \\
H12S20 & 53,1 & 24,7 & 15,9 & 2,15 & 0,64 \\
H12S30 & 55,9 & 20,5 & 19,3 & 2,72 & 0,94 \\
\hline
\end{tabular}

dentre todas as amostras após 28 dias de cura.

A Tabela IX apresenta as composições químicas das amostras após 28 dias de cura, que exibiram variações, principalmente na concentração de sódio $(\mathrm{Na})$ na superfície. A solução com a concentração de $\mathrm{Na}_{2} \mathrm{O}$ mais elevada foi a composição H12S0, entretanto, após a ativação, a amostra H12S10 apresentou uma concentração de sódio na sua superfície mais elevada. Tal fato pode estar relacionado com processos de difusão e eflorescência que ocorreram após a ativação da $\mathrm{CV}$ com a solução alcalina. A rede tridimensional encontrada nos geopolímeros pode ser representada pela fórmula geral $R n-\left[\left(-\mathrm{Si}-\mathrm{O}_{2}\right) z-\mathrm{Al}-\mathrm{O}\right] n \cdot w \mathrm{H}_{2} \mathrm{O}$, onde: $\mathrm{R}$ representa um cátion tal como sódio, potássio ou cálcio; $\mathrm{n}$ representa o grau de policondensação; $z=1,2$ ou 3; e w é a quantidade de água ou grau de hidratação do sistema [8]. A fase presente no gel aluminossilicato foi a responsável pelas propriedades mecânicas do material. Os três tipos de fases mais comumente encontradas em geopolímeros estão listadas na Tabela I. Pela análise de EDS das amostras observou-se que a razão $\mathrm{Si} / \mathrm{Al}$ das amostras variou entre 2,15 e 2,72, demonstrando que a estrutura formada durante a geopolimerização pode ter sido uma variação entre o poli(sialato-siloxo) e poli(sialato-dissiloxo). A razão Na/Al, de acordo com a fórmula geral apresentada acima, deve ser o mais próximo possível de 1,0, visando a obtenção de uma estrutura eletricamente neutra. A amostra H12S30 apresentou valor de $\mathrm{Na} / \mathrm{Al}$ próximo do ideal, demonstrando que a estrutura formada foi perto do balanceamento completo. Tal fato pode corroborar o aparecimento de uma matriz mais estável e que apresente um maior ganho de resistência mecânica, o que de fato foi observado no resultado de resistência à compressão. Com base nos dados apresentados na Tabela IX a estrutura esperada para a amostra H12S30 é a poli(sialato-dissiloxo) e com a maioria dos tetraedros de $\mathrm{AlO}_{4}^{-}$balanceada com íons de $\mathrm{Na}^{+}$provenientes da solução ativadora.

\section{CONCLUSÕES}

Os geopolímeros produzidos a partir da cinza volante apresentaram elevadas resistências mecânicas. As amostras fabricadas com as soluções alcalinas compostas apresentaram melhores resistências mecânicas em todas as idades de cura. Tal resultado indicou que a fonte extra de $\mathrm{SiO}_{2}$ é importante para a obtenção dos geopolímeros. Observou-se que a razão
$\mathrm{SiO}_{2} / \mathrm{Na}_{2} \mathrm{O}$ é importante para a fabricação dos geopolímeros à base de cinza volante. Valores de resistência à compressão próximos de 28 e $48 \mathrm{MPa}$ foram obtidos para as amostras ativadas com a solução contendo razão $\mathrm{SiO}_{2} / \mathrm{Na}_{2} \mathrm{O}$ próximo de 1,0 após 24 h e 28 dias, respectivamente. As amostras ativadas com as soluções compostas apresentaram baixa permeabilidade $\left(0,73 \times 10^{-16} \mathrm{~m}^{2}\right)$, menores valores de absorção de água (12,3\%) e porosidade aparente $(20,1 \%)$ após 28 dias de cura, o que demonstrou que a adição de $\mathrm{Na}_{2} \mathrm{SiO}_{3}$ durante a síntese dos geopolímeros promoveu a obtenção de uma matriz com elevada resistência mecânica e menor valor de porosidade e maior densidade aparente.

\section{AGRADECIMENTOS}

À Universidade Federal de São João del-Rei (UFSJ) e CAPES.

\section{REFERÊNCIAS}

[1] P. Torgal, F.S. Jalali, "A sustentabilidade dos materiais de construção, 2", Univ. Minho, Portugual (2010).

[2] P.S. Deb, P. Nath, P.F. Sarker, Mater. Des. 62 (2014) 32.

[3] R. Davidovits, C. James, Proc. Geopolymer 99, Inst. Géopolymèr, Saint-Quentin, France (1999).

[4] J.L. Provis, S.A. Bernal, Annual Rev. Mater. Res. 44 (2014) 299.

[5] P. Duxson, A. Fernández-Jiménez, J.L. Provis, G.C. Lukey, A. Palomo, J.S.J. van Deventer, J.S. Van Deventer, J. Mater. Sci. 42 (2007) 2917.

[6] J. Davidovits, Geopolymer '88, 1 (1979) 49.

[7] P. Krivenko, "Turn potential into profit, Melbourne" Austrália, Geopolymers (2002) 29.

[8] J. Davidovits, Geopolymer 1 (1988) 25.

[9] J. Davidovits, in: Green chemistry and sustainable development solutions, 1, Inst. Géopolymèr Saint-Quentin França (2005) 9.

[10] M.D.B. Barroso, "Desenvolvimento de compósitos com matriz de geopolímeros reforçados com partículas de quasicristais AlCuFe", Tese Dr., Univ. Fed. Paraíba (2009) 64.

[11] A. Palomo, M.W. Grutzeck, M.T. Blanco, Cem. Concr. Res. 29 (1999) 1323.

[12] O.M. Damilola, Int. J. Mater. Sci. Appl. 2 (2013) 185.

[13] ABNT - Assoc. Brasil. Normas Téc., NBR 5739, 
"Concreto - ensaio de compressão de corpos de prova cilíndricos" (2007).

[14] R.P. Williams, A. van Riessen, Fuel 89 (2010) 3683.

[15] A. Fernández-Jiménez, A. Palomo, Microporous Mesoporous Mater. 86 (2005) 207.

[16] X. Guo, H. Shi, W.A. Dick, Cem. Concr. Compos. 32 (2010) 142.

[17] J.L. Provis, J.S.J. van Deventer, in: Geopolymers: structures, processing, properties and industrial applications, 1 Ed., USA (2009) 15.

[18] F. Škvára, T. Jílek, L. Kopecký, Ceram. - Silikaty 49 (2005) 195.

[19] F. Winnefeld, A. Leemann, M. Lucuk, P. Svoboda, M.
Neuroth, Constr. Build. Mater. 24 (2010) 1086. [20] T. Bakharev, Cem. Concr. Res. 35 (2005) 658.

[21] D. Zaharaki, K. Komnitsas, V. Perdikatsis, J. Mater. Sci. 45 (2010) 2715.

[22] W. Mozgawa, J. Deja, J. Mol. Struct. 924-926 (2009) 434.

[23] V.F.F. Barbosa, K.J.D. MacKenzie, C. Thaumaturgo, Int. J. Inorg. Mater. 2 (2000) 309.

[24] M. Criado, A. Palomo, A. Fernández-Jiménez, Fuel 84 (2005) 2048.

[25] G. Görhan, G. Kürklü, Compos. Part B Eng. 58 (2014) 371.

(Rec. 29/03/2016, Rev. 20/04/2016, 16/06/2016, Ac. $25 / 09 / 2016)$ 